AUTOREN

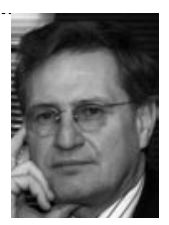

PROF. DR.-ING. HORST FRIEDRICH ist Leiter des DLRInstituts für Fahrzeugkonzepte in Stuttgart.

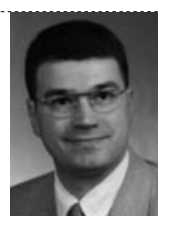

DR.-ING. MICHAEL SCHIER ist Abteilungsleiter der Abteilung Alternative Antriebe und Energiewandlung am DLRInstitut für Fahrzeugkonzepte in Stuttgart.

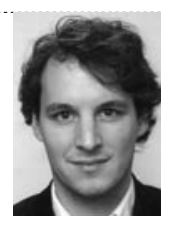

DIPL.-ING. CHRISTIAN HÄFELE ist Projektleiter Thermoelektrik am DLR-Institut

für Fahrzeugkonzepte in Stuttgart.

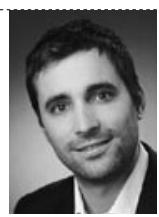

DIPL.-ING. TOBIAS WEILER ist wissenschaftlicher Mitarbeiter am DLRInstitut für Fahrzeug konzepte in Stuttgart.

\title{
STROM AUS ABGASEN - FAHRZEUGGERECHTE ENTWICKLUNG THERMOELEKTRISCHER GENERATOREN
}

Im Zuge der zunehmenden Forderung nach Reduktion des Kraftstoffverbrauchs und $\mathrm{CO}_{2}$-Ausstoßes verbrennungsmotorisch betriebener Fahrzeuge gilt es, bisher ungenutzte Energieflüsse als Nutzenergie zu verwenden. Da hier bekanntermaßen von der zugeführten Energie ein Drittel als Abgaswärme gewandelt wird, macht es Sinn, an der Nutzung dieser Potenziale zu arbeiten: Der Einsatz von thermoelektrischen Generatoren (TEG) erlaubt, Teile der bisher verlorenen Abgasexergie künftig zur Konvertierung in elektrische Energie zu nutzen. Das Deutsche Zentrum für Luft- und Raumfahrt (DLR) hat hierzu fahrzeuggerechte TEG entwickelt, wobei in Zusammenarbeit mit der BMW Group 200 W elektrischer Leistung in einem Versuchsfahrzeug demonstriert wurden. Mit zukünftigen Materialien sind Leistungen von bis zu 600 W und damit ein Verbrauchspotenzial von $5 \%$ zu erwarten. Die aktuellen Forschungsschwerpunkte zielen daraufhin die Fertigungstechnik vor dem Hintergrund eines späteren Serieneinsatzes zu verbessern.

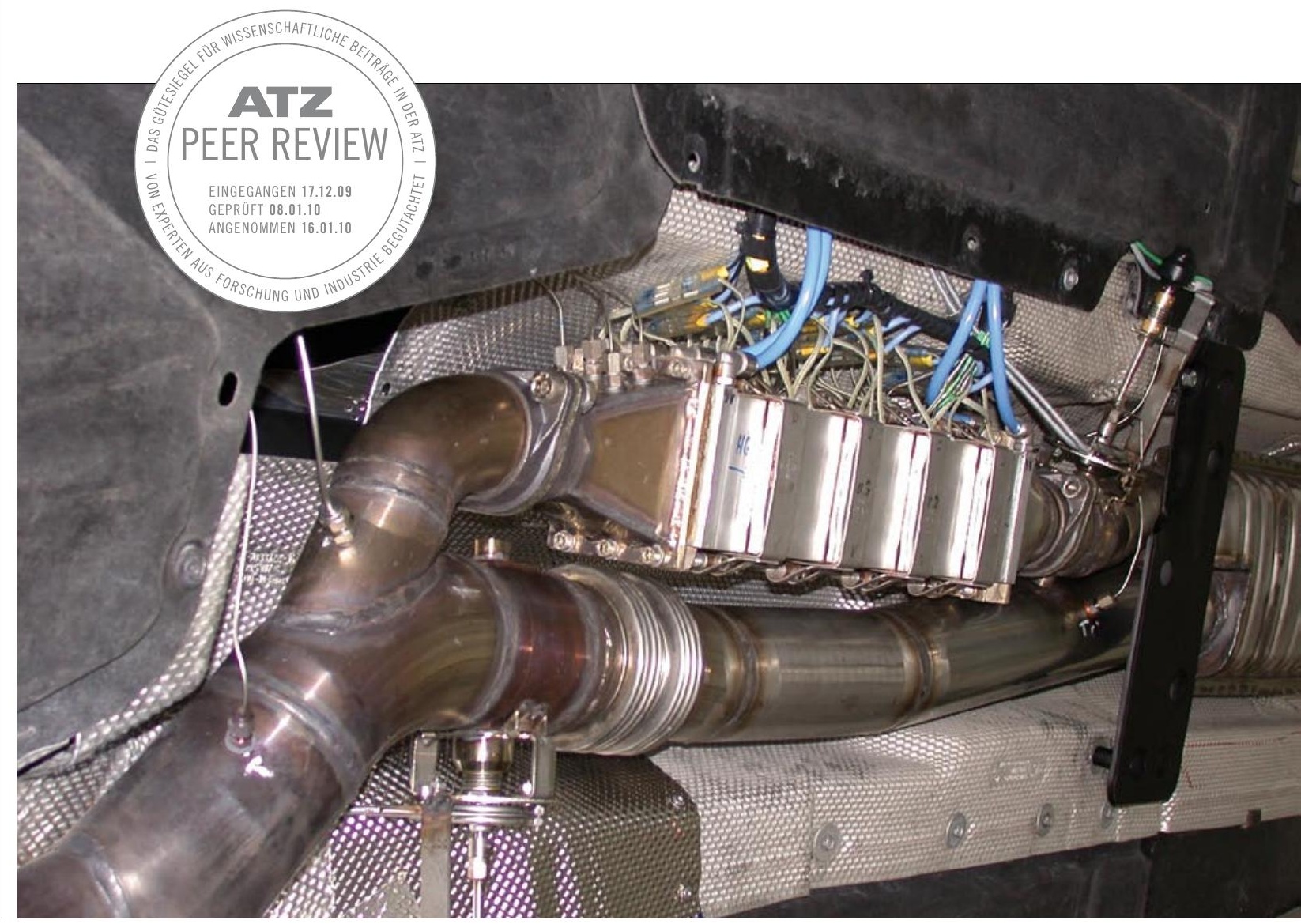


1 EINLEITUNG

2 GRUNDLAGEN

3 EINFLÜSSE AUF DAS FAHRZEUG-GESAMTSYSTEM

4 ENTWICKLUNG FAHRZEUGGERECHTER THERMOELEKTRISCHER GENERATOREN

5 ZUSAMMENFASSUNG UND AUSBLICK

\section{EINLEITUNG}

Die stete Herausforderung zur Reduzierung des $\mathrm{CO}_{2}$-Ausstoßes führt zur Suche nach neuartigen technischen Lösungen für die Automobiltechnik. Die thermoelektrische Energiewandlung stellt dabei eine interessante Möglichkeit dar [1]. Im vorliegenden Artikel werden die Entwicklung fahrzeuggerechter thermoelektrischer $\mathrm{Ge}$ neratoren (TEG) und deren Integration in die Fahrzeugarchitektur beschrieben.

Am Deutschen Zentrum für Luft- und Raumfahrt arbeiten mehrere Institute an der Erforschung der thermoelektrischen Energiewandlung. Das Projekt Sekundärenergienutzung im Fahrzeug wird vom Institut für Fahrzeugkonzepte in Stuttgart koordiniert und bearbeitet. Dort werden die fahrzeugseitigen Anforderungen definiert sowie die Prototypen entwickelt und in die Fahrzeugarchitektur integriert. Das Institut für Werkstoff-Forschung in Köln untersucht, entwickelt und charakterisiert thermoelektrische Materialien und Module, die thermomechanische Spannungsbelastung wird vom Institut für Technische Thermodynamik berechnet. In Zusammenarbeit mit der BMW Group und dem Institut für Werkstoff-Forschung hat das Institut für Fahrzeugkonzepte einen Prototypen eines TEG in ein Fahrzeug des Typs BMW 535i US integriert [2].

Praktische Anwendungen der Thermoelektrizität zeichnen sich unter anderem dadurch aus, dass thermische Energie ohne den Einsatz mechanisch bewegter Teile in elektrische Energie umgewandelt werden kann. Die aus der Raumfahrt als äußerst langlebig bekannte Technik ist es wert, für den Einsatz auch im Fahrzeug untersucht zu werden.

Nach der Analyse der fahrzeugseitigen Anforderungen wurden in den letzten Jahren mehrere TEG-Generationen prototypisch entwickelt, aufgebaut und getestet. Ziel der Darstellung ist die Untersuchung etwaiger Auswirkungen der thermoelektrischen Energiewandlung auf das Gesamtfahrzeug. Das rechnerische Potenzial zur Verbrauchsreduzierung liegt im Prozentbereich [2].

Das DLR besitzt Prüfstände zur Ermittlung der thermoelektrischen Eigenschaften in Köln, für den TEG steht in Stuttgart ein Heißgasprüfstand sowie für die Untersuchung im Fahrzeug ein Klimarollenprüfstand bereit. Forschungsfahrzeuge für Messungen und Einbau von Komponenten sind ebenfalls vorhanden.

\section{GRUNDLAGEN}

\subsection{THERMOELEKTRISCHE ENERGIEWANDLUNG}

Die Wandlung von thermischer Energie in elektrische Energie basiert auf dem im Jahr 1821 von Thomas Seebeck entdeckten Effekt [3]. Er besagt, dass an einer Leiterpaarung aus unterschiedlichen Materialien eine elektrische Spannung entsteht, wenn diese einer Temperaturdifferenz $\Delta T=T_{\mathrm{h}}-T_{\mathrm{c}}$ ausgesetzt wird. Umgekehrt kann eine solche Leiterpaarung aber auch als Wärmepumpe betrieben werden, wenn ein elektrischer Strom durch sie geleitet wird (Peltier-Effekt).

Die diesen Effekten zugrunde liegenden physikalischen Grundlagen und mathematischen Gleichungen wurden bereits in einem MTZ-Beitrag [2] ausführlich beschrieben. An dieser Stelle soll lediglich noch einmal auf die für die Praxis wichtigsten Zusammenhänge eingegangen werden. Die thermoelektrisch erzeugte Leistung $P_{\mathrm{el}}$ ist definiert als:

$$
\text { GL. } 1 \quad P_{e l}=\eta_{\text {TE }} \cdot \dot{Q}
$$

Dabei beschreibt $Q$ den heißseitig eintretenden Wärmestrom, $\eta_{\mathrm{TE}}$ den Wirkungsgrad des thermoelektrischen Materials und $T_{\mathrm{h}}-T_{\mathrm{c}}$ die anliegende Temperaturdifferenz. Für das Leistungsmaximum $P_{\text {el max }}$ kann der Wirkungsgrad geschrieben werden als [4]:

$$
\text { GL. } 2 \quad \eta_{T E}\left(@ P_{e l \max }\right)=\underbrace{\frac{T_{h}-T_{c}}{T_{h}} \cdot \frac{1}{\frac{4}{Z T}+2-\frac{1}{2} \cdot \frac{T_{h}-T_{c}}{T_{h}}}}_{\eta_{c}}
$$

Hierin ist $Z T$ eine dimensionslose Gütezahl, welche eingeführt wurde, um thermoelektrische Materialien bezüglich Ihrer Materialkennwerte zu charakterisieren. Es wird deutlich, dass die elektrische Leistung mit steigendem eintretendem Wärmestrom, höheren $Z T$-Werten und zunehmender Temperaturdifferenz steigt.

Die Entwicklung der ZT-Werte ist vielversprechend. Während bei den bislang gängigen Materialien wie Bismuttellurid $\left(\mathrm{Bi}_{2} \mathrm{Te}_{3}\right)$ und Bleitellurid ( $\mathrm{PbTe}$ ) ZT-Werte um 1 erreicht werden, sind bei neueren, kostengünstigeren Materialien wie Siliziden, Half-Heusler-Materialien und vor allem Skutteruditen $Z T$-Werte von 1,3 realistisch. Technisch dargestellt werden die thermoelektrischen Materialien in plättchenförmigen thermoelektrischen Modulen (TEM), in welchen eine Vielzahl $n$ - und p-dotierter Thermoschenkel wechselweise in Reihe geschaltet werden,

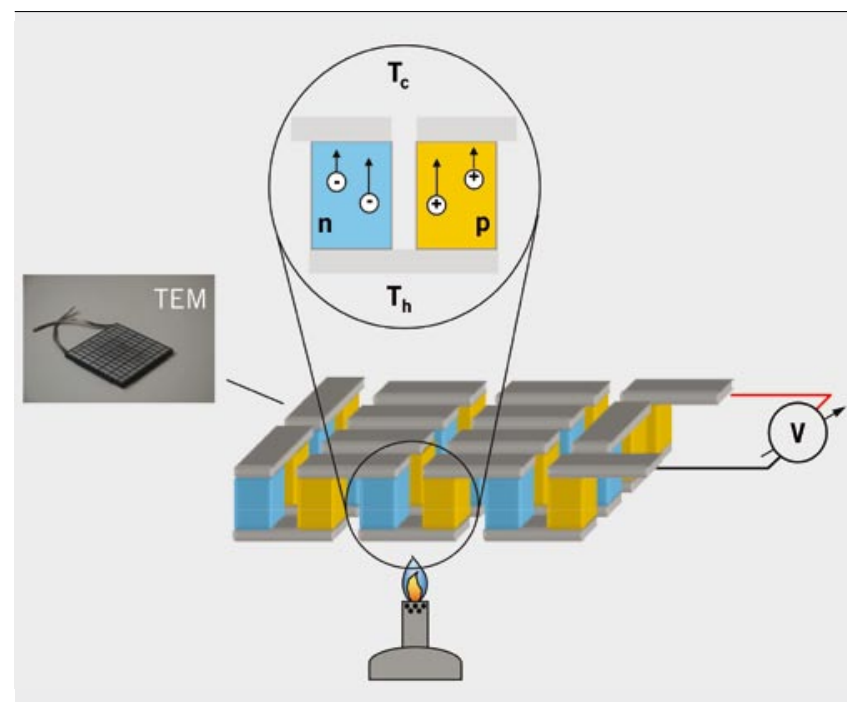

(1) Prinzipdarstellung eines thermoelektrischen Moduls (TEM) und seiner dotierten Halbleiterschenkel [5] 


\subsection{FAHRZEUGRANDBEDINGUNGEN}

Wie bereits erläutert, hängt die Leistungsfähigkeit des TEG in hohem Maß von der anliegenden Temperaturdifferenz sowie dem zugeführten Wärmestrom ab, weshalb Einbauorte mit hohem Exergiepotenzial zu bevorzugen sind. Aus diesem Grund konzentrieren sich die Forschungsarbeiten im Hinblick auf eine automobile Anwendung schwerpunktmäßig auf die TEG-Integration in die Abgasanlage. Allerdings stellen die hier herrschenden Fahrzeugrandbedingungen auch hohe Anforderungen an den TEG. Zur hohen thermischen Belastung der Bauteile kommen korrosive Einflüsse durch aggressives Abgaskondensat von innen sowie Salzlösungen(Tausalz) von außen. Aber auch Schläge, Erschütterungen und Schwingungen, die in Fahrzeugabgasanlagen auftreten, dürfen den TEG nicht beeinträchtigen und müssen bei der Entwicklung berücksichtigt werden.

\section{EINFLÜSSE AUF DAS FAHRZEUGGESAMTSYSTEM}

Eine große Herausforderung stellt sich in der Frage, welche Auswirkungen der Einbau eines TEG auf das Gesamtfahrzeug hat. Zum einen erhöht sich das Fahrzeuggewicht, zum anderen muss der Teil des Wärmestroms, der nicht in elektrische Energie gewandelt wird an die Umgebung abgegeben werden. Der verursachte Gegendruck im Abgasstrang muss minimiert werden, um Gaswechsel und Ausschiebearbeit nicht negativ zu beeinflussen. Außerdem darf die Wärmeentnahme die folgenden Abgasnachbehandlungssysteme nicht beeinträchtigen. Zur Einspeisung der elektrischen Leistung in das Bordnetz werden weitere Komponenten benötigt.

Für die Abgabe der Wärmeleistung an die Umgebung stehen prinzipiell zwei Möglichkeiten bereit. Das vorhandene Kühlsystem kann die zusätzliche Wärmeleistung aufnehmen und an die Umgebung abgeben, solange es nicht an der Auslastungsgrenze ar-

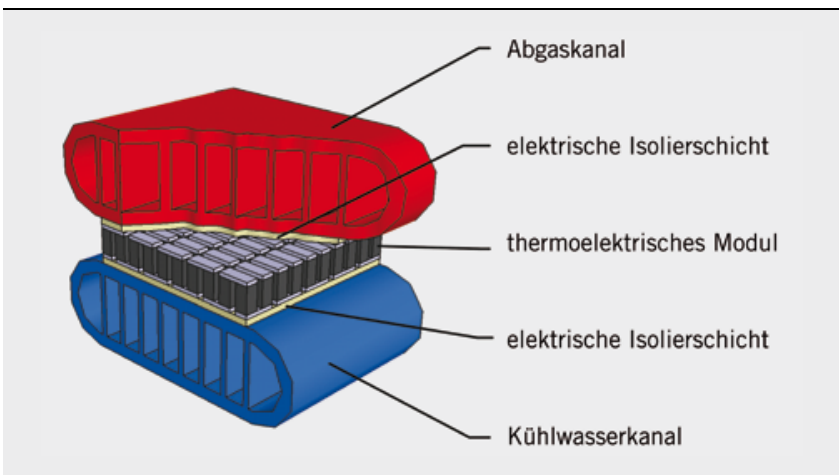

2 Funktionale Schicht eines thermoelektrischen Generators mit Wärmeübertragern für Abgas und Kühlwasser, thermoelektrischem Modul (TEM) und elektrischer Isolierung

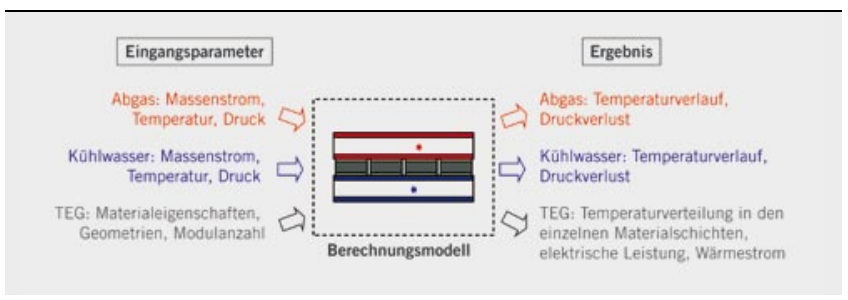

3 Eingangsparameter und Ergebniswerte des TEG-Berechnungsmodells beitet, was aber nur in sehr seltenen Fahrzuständen, beispielsweise bei Motorvolllast an heißen Sommertagen, der Fall ist. In diesen Fällen kann der Abgasmassenstrom über einen Bypass am TEG vorbeigeführt werden. Eine Vergrößerung der Kühlerfläche würde sich in einer Erhöhung des Luftwiderstandes auswirken und sollte nach Möglichkeit vermieden werden.

Vorteilhaft ist in jedem Fall die schnellere Aufheizung des Kühlkreislaufes. Durch die eingetragene Abgaswärme verkürzt sich der Motorwarmlauf, was zu einer reduzierten Motorreibung sowie einem gesteigerten Komfort durch die schnellere Erwärmung der Fahrgastzelle führt.

Bei der Wahl des Einbauorts in der Abgasanlage muss streng darauf geachtet werden, dass der Warmlauf der Abgasnachbehandlungskomponenten nicht negativ beeinträchtigt wird, wobei hier unterschiedliche Strategien denkbar sind. Zum einen kann der TEG hinter den entsprechenden Systemen eingebaut werden, mit dem Nachteil, dass hier deutlich weniger Exergie zur Verfügung steht und somit die Leistungsfähigkeit des TEG reduziert wird. Auf der anderen Seite muss bei einem Einbau vor den Abgasnachbehandlungskomponenten durch entsprechende Maßnahmen dafür gesorgt werden, dass ein schnelles Anspringen der Abgasnachbehandlung gewährleistet ist. Dies kann beispielsweise durch ein Bypasssystem gelöst werden. Auch können die TEM im „Peltier“Betrieb das Aufheizen des TEG unterstützen.

Die Auslegung des Wärmeübertragers stellt höchste Ansprüche an eine gleichmäßige Wärmeverteilung. Die TEM müssen mit der höchstzulässigen Temperatur beaufschlagt werden. Dabei sollte nach Möglichkeit keines davon in einem abweichenden Betriebspunkt betrieben werden, da dieses einzelne sonst den Wirkungsgrad des gesamten Generators beeinträchtigt. Eine als optimal zu bezeichnende Auslegung des Wärmeübertragers wird also nicht für jeden Massestrom gültig sein. Die elektronische Anbindung einzelner Modulgruppen kann hier unterstützend wirken, wenn sie Ungleichmäßigkeiten in der Temperaturverteilung durch einzelne Modul-Impedanzanpassungen ausgleicht. Wie im Bereich der Photovoltaik-Wandlertechnik sind hier DC/DC-Wandler vorzusehen, die den Lastwiderstand - entsprechend der anteiligen Leistungsentnahme aus dem Bordnetz - an den momentan wirkenden elektrischen Innenwiderstand der Module anpassen.

\section{ENTWICKLUNG FAHRZEUGGERECHTER THERMOELEKTRISCHER GENERATOREN}

\subsection{TEG-SIMULATIONSMODELLE}

Um eine Aussage über die zu erwartende elektrische Leistung sowie den verursachten Abgasgegendruck zu ermöglichen, werden zu Beginn einer Entwicklung wärmetechnische Simulationsrechnungen durchgeführt. Hierfür wurde am DLR mit Hilfe der Software EES [6] ein diskretisiertes Zellenmodell des TEG erstellt, wobei bestehende geometrische Symmetrien genutzt und eine funktionale TEG-Schicht, 2, modelliert wurde. Diese besteht aus einer Hälfte eines Abgaswärmeübertragers, einer Hälfte eines Kühlmittelwärmeübertragers und dazwischen einer Schicht von TEMs mit den zugehörigen Isolationsschichten. Dabei wurde der beschriebene Aufbau in viele Einzelzellen unterteilt und für jede Zelle anhand der Energiebilanzen sowie der Stoff- und Wärmetransportbeziehungen ein Gleichungssystem aufgestellt und mit der Nachbarzelle in Beziehung gesetzt. 


\section{Erstmals Firmen-Know-how in Buchform, für den Antriebsstrang moderner Pkws}

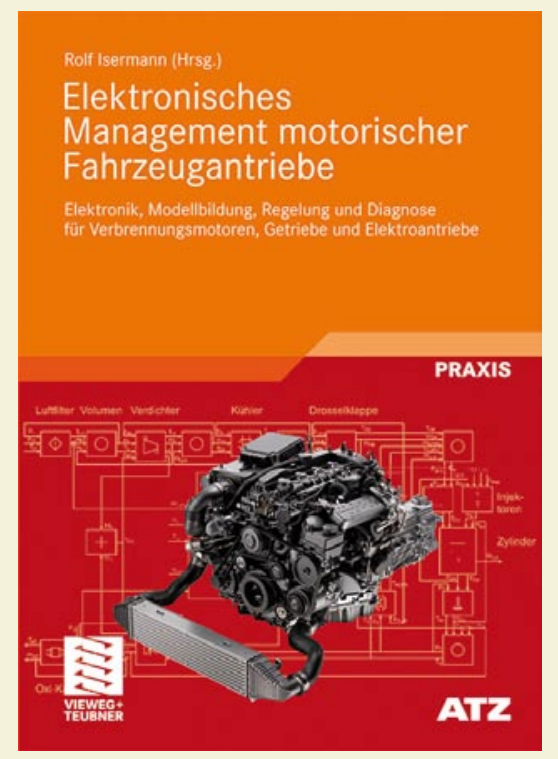

Ja, ich bestelle

Exemplare

Elektronisches Management motorischer Fahrzeugantriebe ISBN 978-3-8348-0855-4 EUR 49,95

Änderungen vorbehalten. Erhältlich im Buchhandel oder beim Verlag. Innerhalb Deutschlands liefern wir versandkostenfrei.

Geschäftsführer: Dr. Ralf Birkelbach (Vors.), Armin Gross, Albrecht F. Schirmacher. AG Wiesbaden HRB 9754

Wenn Sie von uns keine Werbung erhalten möchten, senden Sie uns bitte ein eMail an: widerspruch.springerfachmedienwiesbaden@springer.com
Rolf Isermann (Hrsg.)

Elektronisches Management motorischer Fahrzeugantriebe

Elektronik, Modellbildung, Regelung und Diagnose für Verbrennungsmotoren, Getriebe und Elektroantriebe

2010. XVI, 462 S. mit 318 Abb. und 24 Tab. (ATZ/MTZ-Fachbuch) Geb. EUR 49,95 ISBN 978-3-8348-0855-4

Die stark gestiegenen Forderungen zur Erhöhung der Leistung und zur Senkung von Kraftstoffverbrauch und Emissionen führen zu einer Zunahme der Steuerungs-, Regelungsund Diagnosefunktionen. Dieses Buch zeigt Entwurf, Erprobung und Implementierung dieser elektronischen Managementfunktionen. An verschiedenen Beispielen werden der modellgestützte Entwurf der Steuerung und Regelung von Otto- und Dieselmotoren und ihre Applikation im Detail beschrieben, von der Modellbildung bis zur Brennraumdruck-Regelung. Es zeigt das systematische Vorgehen, umfassende Modellbildungs- und Simulationstools und effiziente Applikationsmethoden.

Der Herausgeber

Prof. Dr.-Ing. Rolf Isermann leitete zuletzt das Fachgebiet Regelungstechnik und Prozessautomatisierung im Institut für Automatisierungstechnik an der TU Darmstadt. Nach seiner Emeritierung im September 2006 leitet er die Forschungsgruppe Regelungstechnik und Prozessautomatisierung.

Fax $+49(0) 611.7878-420$

Firma

Name, Vorname 32110001 Abteilung 


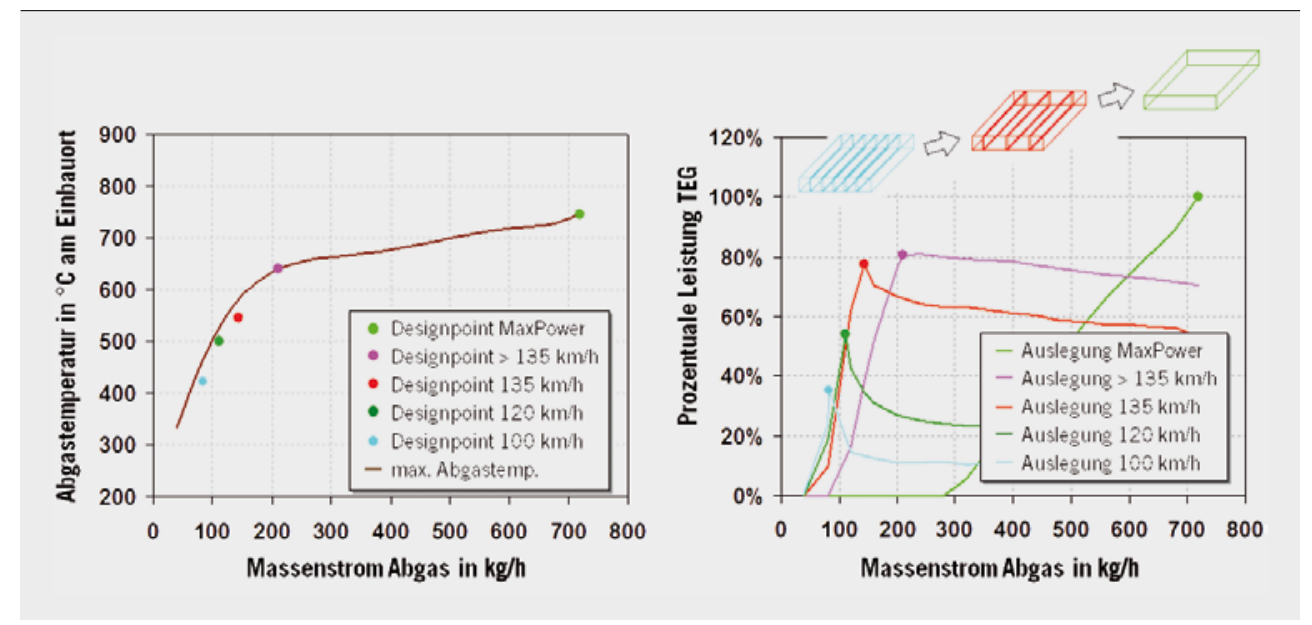

(4) Lage der Designpoints im Abgastemperatur-Abgasmassenstrom-Feld (links); simulierte TEG-Leistungskurven für auf unterschiedliche Lastzustände (Designpoints) optimierte TEG-Designs (rechts)
Zusätzlich sind die Materialdaten der TEM, welche durch Messungen am Institut für Werkstoff-Forschung ermittelt wurden, im Modell hinterlegt. Das Gesamtgleichungssystem kann anschlieBend unter Vorgabe der thermodynamischen Randbedingungen Massenstrom, Temperatur und Druck für Abgas und Kühlwasser iterativ gelöst werden, woraus sich als Ergebnisse die Temperaturverläufe der ins Kühlsystem eingetragene Wärmestrom sowie die elektrische Leistung des TEG ergeben. Auch die Gegendrücke welche durch die wärmeübertragenden Strukturen in den Medienströmen verursacht werden, werden über entsprechende Korrelationen aus den geometrischen Verhältnissen und der Reynoldszahl berechnet. 3 zeigt eine Zusammenfassung der Eingangsparameter und Ergebniswerte des TEG-Berechnungsmodells. Soll der TEG aus mehreren funktionalen Schichten bestehen, so wird das Modell durch Skalierung entsprechend angepasst.

Bei der Modellierung der wärmeübertragenden Berippung spielen zwei Anforderungen eine entscheidende Rolle. Zum einen wird darauf geachtet, dass am TEM die maximal mögliche Temperaturdifferenz anliegt. Hierbei muss bei den heute verfügbaren $\mathrm{Bi}_{2} \mathrm{Te}_{3}$ Modulen beachtet werden, dass an der Modulheißseite eine Tem- peratur von $250{ }^{\circ} \mathrm{C}$ nicht überschritten wird, weil die Module sonst beschädigt würden. Zum anderen muss die Berippung so gestaltet werden, dass der Abgasgegendruck nicht zu stark ansteigt. Da die Berippung starr ist und im Betrieb des TEG nicht dem hochdynamischen thermodynamischen Randbedingungen des Abgases angepasst werden kann, muss die Auslegung auf einen bestimmten Fahrzustand (Designpoint) optimiert werden. Werden höhere Lastzustände erreicht, so wird zum Schutz der TEM und zur Vermeidung eines zu hohen Abgasgegendrucks ein Teil des Abgasmassenstroms am TEG vorbeigeleitet.

4 zeigt links die Lage einer Auswahl von Designpoints im Abgasmassenstrom-Abgastemperatur-Feld eines Referenzfahrzeugs am potenziellen Einbauort. Die braune Kurve stellt den Verlauf der maximalen Abgastemperatur über dem Massenstrom dar. In (4) ist rechts die elektrische Leistung der zugehörigen TEG-Designs über dem Abgasmassenstrom dargestellt. Die Leistungskurven zeigen einen typischen gipfelförmigen Verlauf. Im unteren Lastbereich steigen die Kurven stark an, bevor im Designpoint die Maximalleistung erreicht wird. Nach Überschreiten des Designpoints wird durch Öffnen des Bypasses der Massenstrom durch den TEG reduziert, wodurch Tem-

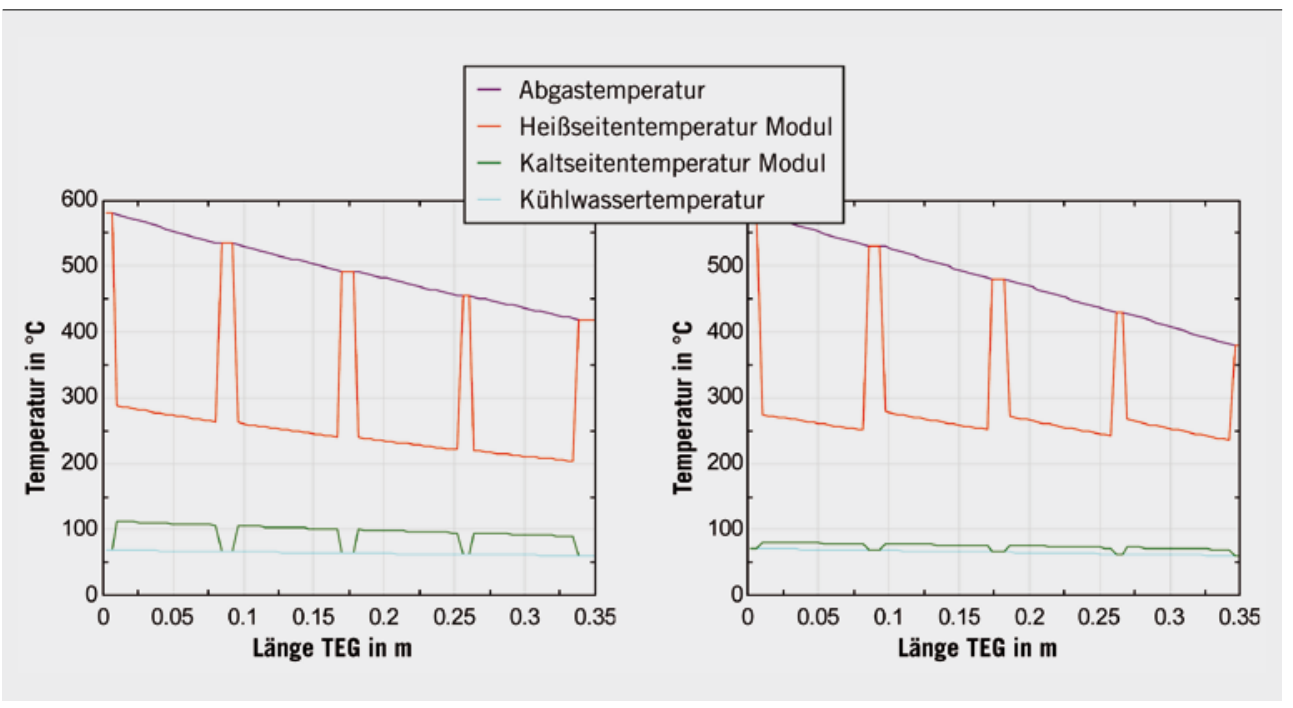

5 Temperaturverläufe über der TEG Längsrichtung für Abgas, Modulheißseite, Modulkaltseite und Kühlwasser; konventionelles Design (links) und Design mit abgas- und kühlwasserseitig optimierter Wärmeübertragung (rechts) 
6 Berechnete Leistungsverläufe im Artemis-Autobahn-Zyklus unter Verwendung von unterschiedlichen thermoelektrischen Materialien und entsprechend angepassten Wärmeübertrager-Designs; die Nettoleistung (blau) errechnet sich aus der TEGLeistung (grün) abzüglich der zusätzlich aufgewendeten Leistung von Wasserpumpe (rot) und Kühlerlüfter (umgerechnet auf Motorleistung); dargestellt sind die Leistungsverläufe für die Verwendung von Bismuttelurid (links) und Bleitellurid (rechts) als thermoelektrisches Material [9]
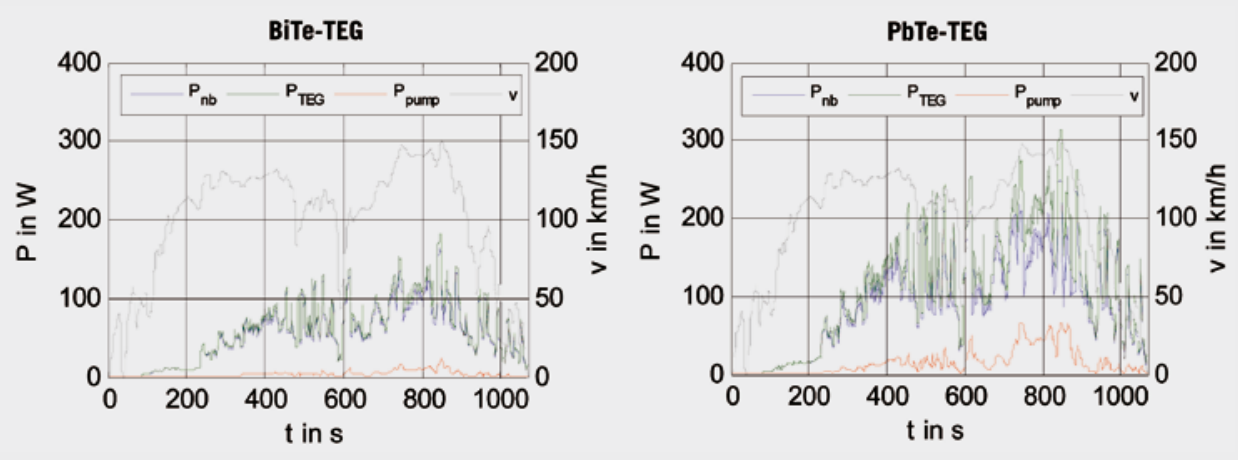

peraturdifferenz und Leistung am TEG abfallen. Im Beispiel zeigt die $135-\mathrm{km} / \mathrm{h}$-Sechster-Gang-Variante einen recht vielversprechenden Verlauf: ein gutes Ansprechverhalten im niedrigen Lastbereich, eine hohe Absolutleistung und einen moderaten Abfall oberhalb des Designpoints.

Mit Hilfe des Simulationsmodells kann gezeigt werden, dass erhebliches Potenzial in der Designoptimierung Thermoelektrischer Generatoren steckt. In $\mathbf{5}$ sind die simulierten Temperaturverläufe von Abgas, Modulheißseite, Modulkaltseite und Kühlwasser über der Längsrichtung des TEG für ein konventionelles (links) und ein optimiertes Design (rechts) dargestellt. Das konventionelle Design zeigt infolge des zunehmenden Wärmeentzugs durch den Wärmeübertrager einen recht gleichmäßigen Abfall der Modulheißseitentemperatur in Strömungsrichtung. Die Kaltseitentemperaturen liegen zirka 40 bis $50{ }^{\circ} \mathrm{C}$ über der Temperatur des Kühlwassers. Die berechnete Maximalleistung dieser Variante liegt bei Designpoint bei $195 \mathrm{~W}$, der verursachte Abgasgegendruck bei 23 mbar. In einer Optimierungsschleife wurde anschließend die wärmeübertragende Berippung von Abgas- und Kühlwasserwärmeübertrager optimiert. Dabei wurde der Abgaswärmeübertrager mit einer in Strö- mungsrichtung zunehmenden Rippendichte ausgestattet um dem heißseitigen Temperaturabfall entgegenzuwirken. Der in (5) rechts dargestellte Temperaturverlauf zeigt, dass dadurch die Modulheißseitentemperatur auf einem hohen Niveau gehalten werden kann. Auch kühlwasserseitig wurde die Berippung verbessert, wodurch die Kaltseitentemperatur noch einmal deutlich um 20 bis $30{ }^{\circ} \mathrm{C}$ reduziert werden konnte. Beim Vergleich der beiden Temperaturverläufe erkennt man deutlich die Steigerung der am Modul anliegenden Temperaturdifferenz (Differenz zwischen roter und grüner Kurve) beim optimierten Design. Dies wirkt sich auch entsprechend in der berechneten Leistung aus, die im Vergleich zum konventionellen Design um $50 \%$ auf 300 W gesteigert werden konnte. Infolge des strömungsoptimierten Designs der Berippung steigt der verursachte Abgasgegendruck nur minimal auf 25 mbar.

Neben dem beschriebenen stationären TEG-Modell steht am Institut für Fahrzeugkonzepte ein dynamisches Modelica-Modell [7] zur Verfügung, um auch das transiente Verhalten des TEGs im Zyklus simulieren zu können. Die linke Grafik in 6 zeigt die berechnete Entwicklung der Nettonutzleistung des zuvor beschriebenen 135-km/h-Designs mit $\mathrm{Bi}_{2} \mathrm{Te}_{3}$-Modulen im Artemis-Autobahn-Zyklus 


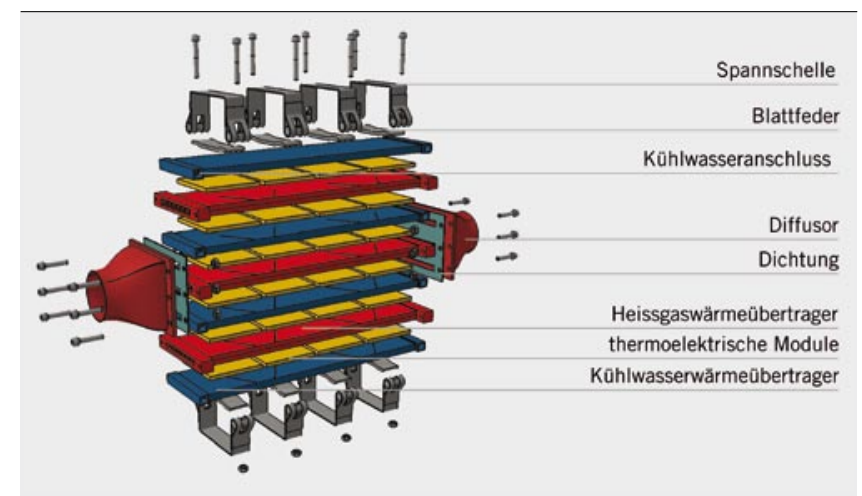

CAD-Explosionsdarstellung eines TEG in Stapelbauweise mit $24 \mathrm{Bi}_{2} \mathrm{Te}_{3}$ Modulen [2]

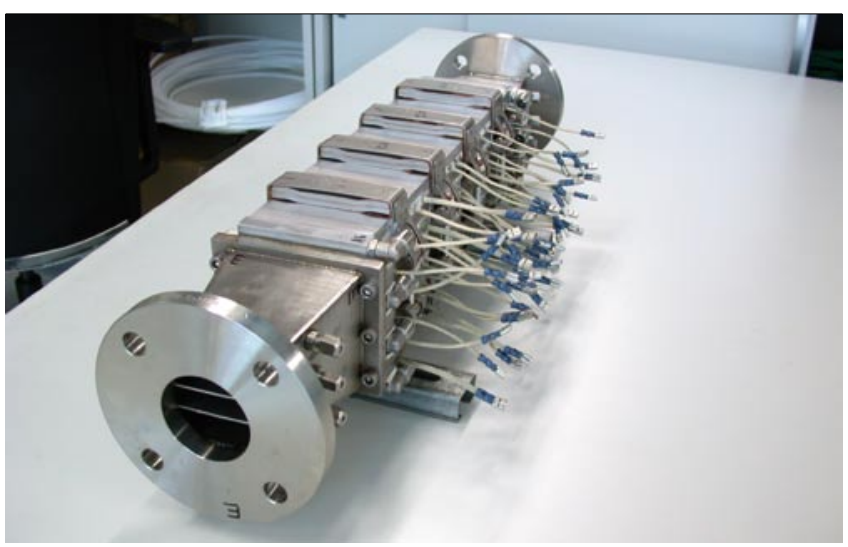

8 TEG-Funktionsmuster mit angeflanschten Prüfstandsdiffusoren

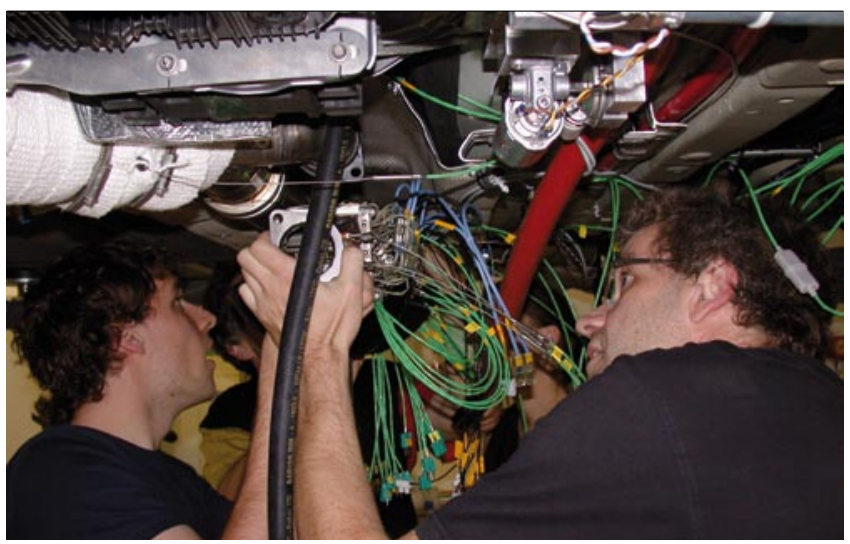

(9) Fahrzeugintegration eines TEG-Funktionsmusters

[8]. Es ist deutlich das Aufheizverhalten des TEG zu beobachten, wobei anzumerken ist, dass die hinterlegten thermischen Massen aus Prototypengeometrien abgeleitet wurden und mit zirka $11 \mathrm{~kg}$ für den TEG noch als relativ hoch angenommen wurden. Im Maximum werden bei dieser Variante $180 \mathrm{~W}$ erreicht.

Um die Potenziale leistungsfähigerer Materialien für den mittleren Temperaturbereich aufzuzeigen, sind in der rechten Grafik von (6) die Leistungsverläufe eines TEG-Systems abgebildet, bei dem im Modell PbTe-Materialdaten hinterlegt wurden. Die wärmeübertra- gende Struktur wurde an die höheren Einsatztemperaturen des Bleitellurid von bis zu $450{ }^{\circ} \mathrm{C}$ angepasst. Hierdurch kann die Warmlaufphase deutlich verkürzt werden. Auch stellen sich infolge des gröBeren übertragenen Wärmestroms und der besseren Wirkungsgrade von Bleitellurid höhere elektrische Leistungen von bis zu $300 \mathrm{~W}$ im Zyklus ein. Die Beeinflussung der Motorleistung infolge des verursachten Abgasgegendrucks wird derzeit in das Modell integriert.

\subsection{WEITERE ENTWICKLUNGSSCHRITTE}

Im weiteren Entwicklungsprozess wird die vielversprechendste Variante dann weiter ausgestaltet. Dabei müssen auf Basis des zur Verfügung stehenden Bauraums kompakte, leichte sowie fertigungsgerechte Lösungen für unterschiedlichste Teilfunktionen des TEG gefunden werden. Auch in dieser Phase wird bei der Entwicklung auf entsprechende Simulationsprogramme zurückgegriffen. So werden mittels FE-Programmen Berechnungen durchgeführt, um die mechanische und thermomechanische Stabilität der Komponente abzusichern oder um die Strömungs- und Temperaturverteilung im TEG zu untersuchen. Zusätzlich werden die Ergebnisse durch experimentelle Untersuchung von Teilkomponenten abgesichert, wofür umfangreiche Prüfanlagen zur Verfügung stehen. Am Ende des Konstruktionsprozesses stehen ein CAD-Modell des TEG, wie es in $\mathbf{7}$ skizziert ist, sowie entsprechende Fertigungszeichnungen zur Verfügung.

Beim Bau der TEG wurde in der Vergangenheit nach Möglichkeit auf Standardkomponenten zurückgegriffen. Sonderbauteile konnten größtenteils in der DLR-eigenen Werkstatt hergestellt werden. Hinsichtlich der thermoelektrischen Wandlermodule wurden bei den bisherigen DLR-Prototypen bevorzugt Module vom Typ HZ-20 der Firma Hi-Z eingesetzt [10]. Diese Module haben Wirkungsgrade von bis zu 3,5\% und zeichnen sich durch eine hohe Robustheit und Langzeitstabilität aus. 8 zeigt ein aufgebautes TEG-Funktionsmuster mit angeflanschten Prüfstandsdiffusoren.

\subsection{FAHRZEUGINTEGRATION}

Wie eingangs bereits erwähnt, wurde ein in Zusammenarbeit mit der BMW Group entwickelter TEG-Prototyp in ein Versuchsfahrzeug integriert. Dabei lagen die Vermessung des Basisfahrzeugs, großteils die hardwaretechnische Umsetzung der Umbaumaßnahmen sowie schließlich die Funktionsgrundprüfung des Fahrzeugs am Klima-Rollenprüfstand im Aufgabenbereich des DLR.

Die Vermessung des Basisfahrzeugs am Rollenprüfstand diente dazu, Erfahrungen mit dem Fahrzeug zu gewinnen und die als Randbedingung für die Auslegung verwendeten Abgastemperaturen abzusichern. In der anschließenden Planungs- und Umbauphase wurden packageoptimierte Lösungen für die Einzelbereiche Abgasanlage, Kühlkreislauf und Messsystem entwickelt und umgesetzt. Nach Einbindung des TEG in die Fahrzeugarchitektur, (9, wurde die Funktion des TEG am Klima-Rollenprüfstand des DLR untersucht.

\subsection{EXPERIMENTELLE UNTERSUCHUNG DER TEG}

Zur Untersuchung der TEG stehen ein leistungsstarker 200-kWGasbrenner, ein Kühlsystem zur Temperierung der TEG-Kaltseite sowie entsprechende Messtechnik und Datenerfassung zur Verfügung. Darüber hinaus können die mit TEG-Prototypen ausgerüsteten Versuchsfahrzeuge auf dem Klima-Rollenprüfstand untersucht werden. 
Vergleich der ermittelten Leistungswerte (links) und Abgasgegendrücke (rechts) für Simulation und Messung

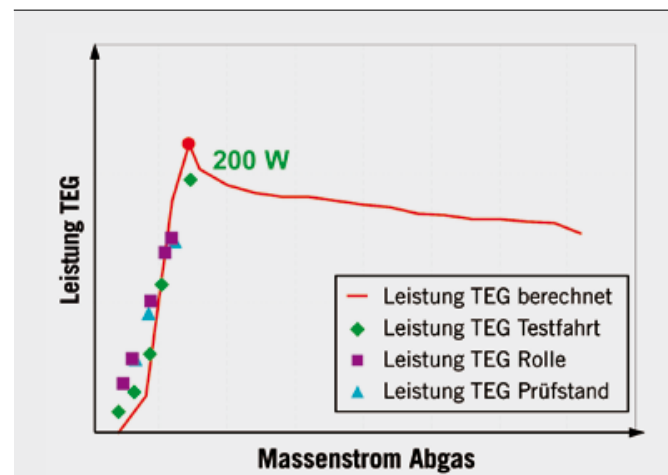

Massenstrom Abgas

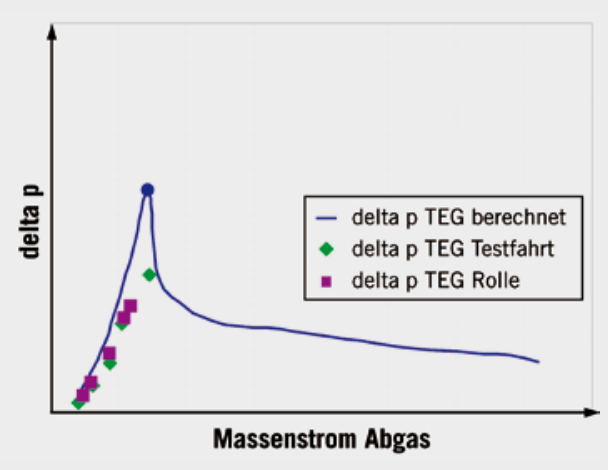

Massenstrom Abgas
(10 zeigt den Vergleich der experimentellen Ergebnisse am Prüfstand und im Versuchsfahrzeug mit den zuvor durchgeführten Simulationen für die oben erläuterte $135 \mathrm{~km} / \mathrm{h}$ Variante. Die Leistungswerte stimmen recht gut mit den Simulationen überein und liegen im Maximum bei 200 W. Der verursachte Abgasgegendruck zeigt deutlich niedrigere Werte als erwartet. Der Designpoint $135 \mathrm{~km} / \mathrm{h}$ konnte nur bei Versuchsfahrten der BMW Group auf einer Teststrecke angefahren werden. Am Heißgasprüfstand stand zum damaligen Entwicklungszeitpunkt noch keine ausreichende Heizleistung zur Verfügung, am Rollenprüfstand stiegen die Temperaturen im TEG-Kühlsystem aufgrund der ungenügenden Anströmung der Kühler zu stark an.

\section{ZUSAMMENFASSUNG UND AUSBLICK}

Die Nutzung der Abgasenthalpie mit Hilfe thermoelektrischer Generatoren (TEG) verbessert die Energieeffizienz von Fahrzeugen mit Verbrennungsmotor. Durch die Integration von thermoelektrischen Modulen in den Abgasstrang und in die Energiearchitektur eines BMW 535i US gelang dem Deutschen Zentrum für Luft- und Raumfahrt (DLR) mit einem Forschungsdemonstrator bei einem TEG-Wirkungsgrad von 2,0\% die Bereitstellung von 200 W elektrischer Leistung. Die künftigen Entwicklungsschwerpunkte sind die Verbesserung des Wirkungsgrades bei neuen beziehungsweise weiterentwickelten thermoelektrischen Materialien, die Optimierung der Wärmeübergänge und die Fahrzeugintegration in weiteren thermodynamisch und betriebstechnisch sinnvollen Bereichen.

Ein weiterer Forschungsschwerpunkt am DLR zielt derzeit darauf, die Fertigungstechnik der Generatoren vor dem Hintergrund eines späteren Serieneinsatzes zu verbessern. Aktuelle Funktionsmuster sind kraftschlüssig verspannte - und damit relativ schwere - Systeme, deren Zusammenbau höchst zeitintensiv ist. Hier wird eine Aufbautechnik entwickelt, die mit serientauglichen - vorzugsweise stoffschlüssigen - Fügeverfahren arbeitet und dabei den hohen betriebstechnischen und Kosten-Anforderungen des Systems gerecht wird.
Die Automobilhersteller prognostizieren für derzeit verfügbare thermoelektrische Materialien mit $Z T$-Werten von 0,85 eine elektrische Leistung von $600 \mathrm{~W}$ beziehungsweise ein Verbrauchspotenzial von $5 \%$ bei Autobahnfahrt [2]. Diese Werte können bei weiterer Steigerung der $Z T$-Werte noch übertroffen werden, vorausgesetzt die darüber hinaus erzeugte Leistung wird von den elektrischen Verbrauchern auch benötigt und abgenommen.

\section{LITERATURHINWEISE}

[1] Friedrich, H. E.; Treffinger, P.; Müller, E.: Management von Sekundärenergie und Energiewandlung von Verlustwärmeströmen. Vortrag, ATZ/MTZ-Konferenz Energie, München, 26. und 27. Juni 2007

[2] Liebl, J.; et al.: Der thermoelektrische Generator von BMW macht Abwärme nutzbar. In: MTZ 70 (2009), Nr. 3, S. 272-281

[3] Seebeck, T. J.: Magnetische Polarisation der Metalle und Erze durch Temperatur-Differenz. In: Abhandlungen der Königlich Preussischen Akademie der Wissenschaften zu Berlin aus den Jahren 1822 und 1823. (1825)

[4] Müller, E.: Thermoelectric Materials for Waste Heat Recovery, Linearized Thermal Sensors and Peltier Cooling - Material and System Development for Device Applications. Vortrag, Steinfurter Keramiktage, FH Münster/Steinfurt, 15. bis 19. Dezember 2002

[5] Weiler, T.; Häfele, Ch.; Schier, M.; Eder, A.: Wärmetechnische Auslegung eines fahrzeugtauglichen thermoelektrischen Generators. Vortrag, 1. Fachtagung Thermische Rekuperation im Kraftfahrzeug. Haus der Technik,

München, 5. und 6. November 2009

[6] URL: http://www.mhhe.com/engcs/mech/ees/

[7] URL: http://www.modelica.org/

[8] URL: http://www.inrets.fr/ur//te/publi-autresactions/fichesresultats/

ficheartemis/road3/method31/Artemis_cycles_report_LTE0411.pdf

[9] Braig, T.; Ungethüm, J.: System-level Modeling of an ICE-powered Vehicle with Thermoelectric Waste-Heat Utilization. Lecture, International Modelica Conference, Como, Italy, 20th to 22nd September 2009

[10] N. N.: HZ-20 Thermoelectric Module. Hi-Z Technology, Inc., San Diego, USA, http://www.hi-z.com/hz20.php at 14th January 2010

DOWNLOAD DES BEITRAGS

www.ATZonline.de

READ THE ENGLISH E-MAGAZINE

order your test issue now: SpringerAutomotive@abo-service.info
Sie wollen sich verändern oder suchen die erste Stelle, dann senden Sie bitte lhre kompletten Bewerbungsunterlagen an:
C L Engineering GmbH, Schönaustr. 11, 65201 Wiesbaden bewerbung@tlengineering.de - www.tlengineering.de

Tel.: 0611-4060616 - Fax: 0611-4060617 